Chronic Obstructive Pulmonary Diseases: Journal of the COPD Foundation COOPD

\author{
Original Research
}

\title{
Black Carbon Content in Airway Macrophages is Associated with Reduced CD80 Expression and Increased Exacerbations in Former Smokers With COPD
}

Vickram Tejwani, MD ${ }^{1}$ Eric Moughames, MD ${ }^{2}$ Karthik Suresh, MD ${ }^{1}$ Shih-En Tang, MD, PhD ${ }^{3}$ Laura G. Mair, $\mathrm{MS}^{2}$ Karina Romero, MD, $\mathrm{MSc}^{2}$ Nirupama Putcha, MD, MHS ${ }^{1}$ Neil E. Alexis, $\mathrm{PhD}^{4}$ Han Woo, $\mathrm{PhD}^{1}$ Franco R. D’Alessio, $\mathrm{MD}^{1^{* *}}$ Nadia N. Hansel, MD, $\mathrm{MPH}^{1^{* *}}$

\section{Abstract}

Background: Chronic obstructive pulmonary disease (COPD) is characterized by recurrent exacerbations. Macrophages play a critical role in immune response and tissue repair in COPD. Airway macrophages (AM) are exposed to environmental exposures which are retained in the cytoplasmic material. Both biomass and particulate matter have been linked to higher AM black carbon. It is unknown if AM black carbon is associated with COPD morbidity and macrophage phenotype.

Methods: Former smokers with COPD were enrolled and sputum induction was performed to obtain airway macrophages. Macrophages underwent black carbon quantification and flow cytometry phenotyping. Health information was obtained the same day as sputum induction and prospective exacerbations were assessed by monthly telephone calls.

Results: We studied 30 former smokers with COPD who had a mean age of 67 years and mean forced expiratory volume in 1 second $\left(\mathrm{FEV}_{1}\right) \%$ predicted of $60.8 \%$. Higher AM black carbon content was associated with increased total exacerbations and severe exacerbations and reduced CD80 expression.

Conclusion: AM black carbon association with respiratory morbidity is largely unexplored and this is the first study to identify association with prospective exacerbations. Macrophages expressed reduced CD80, a surface marker providing costimulatory signals required for development of antigen-specific immune responses. Our findings suggest that reduced CD80 expression is the pathophysiologic mechanism for the association of AM black carbon content and increased exacerbations. Therefore, beyond solely serving as a marker for increased exposures, AM black carbon content may be a predictor of future exacerbations given a macrophage less equipped to respond to an acute infectious exposure.

\footnotetext{
Abbreviations: chronic obstructive pulomonary disease, COPD; airway macrophages, AM; COPD Assessment Test, CAT; modified Medical Research Council scale, mMRC; Ease of Cough and Sputum Clearance score, ECSC; allophycocyanin, APC; interleukin, IL; transforming growth factor, TGF; tumor necrosis factor, TNF; fluorescence minus one, FMO; mean fluorescence intensity, MFI; incidence rate ratio, IRR

Funding Support: This work was supported by grants from the National Heart, Lung, and Blood Institute (F32HL149258-01, V.T.), the Baurenschmidt Award from the Johns Hopkins Eudowood Foundation (V.T.), the Department of Defense (DoDW81XWH-16-1-0510, F.R.D.), the National Institute on Minority Health and Health Disparities of the National Institutes of Health (P50MD010431, N.N.H), the National Institute of Environmental Health Sciences of the National Institutes of Health (R01ES022607, N.N.H), and the U.S.Environmental Protection Agency (agreement number 83615001, N.N.H.). This manuscript has not been formally reviewed by the Environmental Protection Agency or the National Institutes of Heath, and the views expressed in this document are solely those of the authors and do not necessarily reflect those of the Environmental Protection Agency or the National Institutes of Health.

Date of Acceptance: September 18, 2020 | Published Online Date: November 6, 2020

Citation: Tejwani V, Moughames E, Suresh K, et al. Black carbon content in airway macrophages is associated with reduced CD80 expression and increased exacerbations in former smokers with COPD. Chronic Obstr Pulm Dis. 2021;8(1):91-99. doi: https://doi.org/10.15326/ jcopdf.2020.0170
} 
1 Division of Pulmonary and Critical Care Medicine, Johns Hopkins University, Baltimore, Maryland, United States

2 Johns Hopkins Bayview Medical Center, Baltimore, Maryland, United States

3 Division of Pulmonary and Critical Care Medicine, Tri-Service General Hospital, Taipei, Taiwan

4 Department of Pediatrics, University of North Carolina, Chapel Hill, North Carolina, United States

** These authors share senior authorship

\section{Address correspondence to:}

Vickram Tejwani, MD

Email:tejwani@jhmi.edu

Phone: (443)287-3345

\section{Keywords:}

macrophage; CD80; black carbon; chronic obstructive pulmonary disease; $\mathrm{COPD}$

\section{This article contains an online supplement.}

\section{Background}

Chronic obstructive pulmonary disease (COPD), the third leading cause of death worldwide, ${ }^{1}$ is characterized by recurrent exacerbations and progressive decline in lung function. Macrophages play a critical role in immune response and eventually tissue repair in COPD. ${ }^{2-4}$ Macrophages from COPD patients compared to healthy controls exhibit reduced expression of surface markers important for immunity (adhesion molecules, antigen presentation molecules and recognition markers), as well as alterations in cytokine production. 5,6 Airway macrophages (AMs) are also exposed to environmental exposures, such as cigarette smoke and pollution. ${ }^{7}$

In developed countries, the most strongly supported cause of COPD is cigarette smoking; however, there is growing evidence that environmental exposures other than tobacco smoke, including indoor and outdoor air pollution, are linked to pulmonary morbidity and mortality. ${ }^{8,9}$ Inhaled fine particulate matter avoids upper airway mucociliary trapping and can reach the lower airways where AMs reside. Subsequently, these particles are phagocytosed by AMs and retained in the cytoplasmic material. Both biomass and particulate matter exposure have been linked to higher AM black carbon. ${ }^{10,11}$ Whether black carbon content within AM influences COPD morbidity or macrophage phenotype is yet to be elucidated, therefore, we sought to determine the relationship of black carbon content to COPD morbidity and macrophage phenotype.

\section{Methods}

\section{Participant Recruitment and Macrophage Carbon Assessment}

Former smokers with COPD were enrolled ( $\mathrm{n}=30)$ after meeting the following inclusion criteria: age $\geq 40$ years, post bronchodilator $35 \%<$ forced expiratory volume in 1 second $\left(\mathrm{FEV}_{1}\right) \leq 80 \%$ predicted, $\mathrm{FEV}_{1}$ to forced vital capacity (FVC) ratio $<70 \%$, and $>10$ pack years smoking, with quit date of $>6$ months prior to enrollment and exhaled carbon monoxide level $\leq 6 \mathrm{ppm}$. Participants with evidence of active infection or exacerbation within 2 weeks of study visit did not undergo sputum induction and were excluded from our study. Participants were followed longitudinally at baseline, 3 months and 6 months with comprehensive phenotyping and induced sputum collection. Informed written consent was obtained from all participants, all experiments were performed in accordance with relevant guidelines and regulations, and the study was approved under the Johns Hopkins Institutional Review Board protocol number 00069904.

Sputum induction was performed according to the methods of Alexis et al. ${ }^{12}$ Briefly, the Aerosol Universal III nebulizer was used and sputum samples solubilized with Sputolysin and cytocentrifugation and cytospin slide generated with Diff-Quick for cell differentials. The pellet was re-suspended in freezing media and cryopreserved for future analysis. Digital color images of macrophages on cytospin slides were taken using the Nikon Eyepiece microscope camera (Nikon, Tokyo, Japan) at 100x magnification with oil immersion. Per sample, a total of 50 randomly selected macrophages with an intact cell wall were analyzed for black carbon content using ImageJ software and the mean carbon black area of macrophages was used for analysis. We also counted the fraction of the 50 macrophages that were positive for any black carbon and generated an independent variable of percentage of black carbon positive macrophages for a secondary analysis.

Health information was collected the same day as the sputum collection; specifically, the COPD Assessment Test (CAT), ${ }^{13}$ the modified Medical Research Council (mMRC) Dyspnea Scale ${ }^{14}$ and the Ease of Cough and Sputum Clearance (ECSC) score. Participants were also queried regarding their employment status and if currently working, asked 
if their current job exposed them to "vapors, gas, dust or fumes." Patients were queried regarding the occurrence of mild, moderate or severe exacerbations via monthly telephone calls. Mild, moderate and severe exacerbations were defined as worsening of symptoms leading to an increase in inhaler use without antibiotics or systemic glucocorticoids, exacerbation leading to treatment with antibiotics or systemic glucocorticoids and exacerbation resulting in an urgent care visit, emergency department visit or hospitalization, respectively. Spirometry was performed according to American Thoracic Society criteria. $^{15}$

\section{Macrophage Phenotyping by Flow Cytometry}

The first available sputum sample with at least $1 \times 10^{6}$ cells $(n=16)$ was processed for flow cytometry. Cells were thawed and plated prior to incubation with Golgi Stop and Golgi Plug for 3 hours, then stained with Fixable UV Live/Dead, and then Fc blocked (BD). We stained with the surface markers BV515conjugated anti-CD3 (UCHT1, BD Horizon), BB700conjugated anti-CD19 (SJ25C1, BD Horizon), allophycocyanin (APC)-Cy7-conjugated anti-HLADR (G46-6, BD Pharmingen), BUV395-conjugated anti-CD16 (3G8, BD Horizon) and BUV737conjugated anti-CD 14 (M5E2, BD Horizon), Pe-Cy7conjugated anti-CD80 (L307.4, BD Pharmingen) and APC-R700-conjugated anti-CD274 (MIH1, BD Horizon). Cells were subsequently fixed and permeabilized for 30 minutes and intracellular cytokine staining performed for 30 minutes followed by intracellular staining with APC-conjugated anti-interleukin (IL)-10 (JES3-19F1), BV421conjugated anti-IL-1 $\beta$ (H1b-98, BioLegend), BV510-conjugated anti-IL-8 (G265-8, BD Horizon), PE-CF594-conjugated anti-transforming growth factor(TGF)- $\beta 1$ (TW4-9E7), and BV605-conjugated anti-tumor necrosis factor (TNF)-a (MAb11, BioLegend). Fluorescence minus one (FMO) was utilized to determine specificity for intracellular cytokine expression. Compensation was completed with UltraComp eBeads (eBioscience). Acquisition is performed using a FACSAria instrument with FACSDiva software (BD) and FlowJo version 10.5.0 (Tree Star, Inc., Ashland, Oregon) for analysis. Flow cytometer gating strategy was as follows: single cells were identified first, subsequently, a UV excitable live dead discrimination assay (Invitrogen) was applied. Live cells were excluded for CD3+ and CD19+ populations to remove $\mathrm{T}$ - and $\mathrm{B}$-cells, respectively. After removing these other cell types, we characterized airway macrophages by gating those with characteristic side-scatter and forward-scatter properties and with CD14 positivity (Supplementary Figure 1 in the online supplement). ${ }^{16}$ We then measured the mean fluorescence intensity (MFI) of each surface marker (HLA-DR, CD274 and CD80) and intracellular cytokines (IL-10, IL-1 $\beta$, IL-8, TGF- $\beta 1$, and TNF- $\alpha$ ).

\section{Analysis}

Descriptive analyses were used to examine means and standard deviations or proportions and counts. We performed cross-sectional regression analysis using the data from the participant's earliest visit for which he/she had induced sputum collection. Linear regression analyses were run to evaluate the relationship between AM black carbon content and COPD outcomes, and Poisson regression analyses for exacerbation risk. Models were adjusted for gender and inhaled corticosteroid use, defined as potential confounders based on their bivariate associations $(p<0.2)$ with AM black carbon and any measured outcomes. Other potential confounders including total pack-years smoking and time since smoking cessation were considered but not included given they did not meet the criteria of $p<0.2$ bivariate association. Poisson regressions were performed taking into account follow-up days as offset and using robust standard error to account for potential overdispersion. For immune markers, given their smaller sample sizes and greater susceptibility to outlier influence, an additional analysis using Spearman's rank correlation was conducted to test associations with AM black carbon. Statistical significance was defined as $P<0.05$, and, for immune marker analysis, Bonferroni-corrected $P$-value was evaluated for multiple-comparisons. Analyses were performed with StataSE statistical software and R.

\section{Results}

\section{Participant Characteristics}

Participants (Table 1) had a mean $( \pm S D$ ) age of 67 $( \pm 9.0)$ years, mean $( \pm S D)$ pack years of smoking of 51.7 ( \pm 36.9$)$ with a mean $( \pm S D)$ of $153( \pm 136.9)$ months since quit smoking, and a post-bronchodilator 


\section{Table 1. Participant Characteristics}

\begin{tabular}{|c|c|c|c|}
\hline \multirow[t]{2}{*}{ All Participant Characteristics $(n=30)$} & & $\begin{array}{l}\text { Characteristics of Subset } \\
\text { with Exacerbation }(n=14)\end{array}$ & $\begin{array}{l}\text { Characteristics of Subset } \\
\text { with Adequate Cells for } \\
\text { Flow Cytometry }(n=16)\end{array}$ \\
\hline & \multicolumn{3}{|c|}{ Mean \pm SD or $\%(n)$} \\
\hline Age & $67.0 \pm 9.0$ & $67.5 \pm 8.6$ & $67.6 \pm 10.3$ \\
\hline Female & $53 \%(16)$ & $57 \%(8)$ & $69 \%(11)$ \\
\hline Black & $47 \%(14)$ & $43 \%(6)$ & $38 \%(6)$ \\
\hline \multicolumn{4}{|l|}{ Education } \\
\hline Some college or above & $60 \%(18)$ & $71 \%(10)$ & $50 \%(8)$ \\
\hline \multicolumn{4}{|l|}{ Occupational Status } \\
\hline Employed & $20 \%(6)$ & $21 \%(3)$ & $17 \%(1)$ \\
\hline $\begin{array}{l}\text { Current occupational vapor, } \\
\text { gas, dust or fume exposure }\end{array}$ & $6.7 \%(2)$ & $0 \%(0)$ & $0 \%(0)$ \\
\hline Unable to work & $20 \%(6)$ & $14 \%(2)$ & $19 \%(3)$ \\
\hline Retired & $43 \%(13)$ & $43 \%(6)$ & $50 \%(8)$ \\
\hline Unknown & $17 \%(5)$ & $21 \%(3)$ & $25 \%(4)$ \\
\hline BMI & $32.1 \pm 6.3$ & $30.6 \pm 5.1$ & $31.7 \pm 6.1$ \\
\hline Normal & $13 \%(4)$ & $14 \%(2)$ & $19 \%(3)$ \\
\hline Overweight & $27 \%(8)$ & $36 \%(5)$ & $25 \%(4)$ \\
\hline Obese & $60 \%(18)$ & $50 \%(7)$ & $56 \%(9)$ \\
\hline Total Pack Years & $51.7 \pm 36.9$ & $51.3 \pm 34.8$ & $53.4 \pm 40.9$ \\
\hline Time Since Quit Smoking (months) & $153 \pm 136.9$ & $151.3 \pm 147.2$ & $158.8 \pm 126.2$ \\
\hline Use of ICS, (n) & $53 \%(16)$ & $43 \%(6)$ & $50 \%(8)$ \\
\hline FEV $_{1} \%$ Predicted & $62.1 \pm 11.0$ & $61.7 \pm 10.2$ & $61.0 \pm 12.1$ \\
\hline \multicolumn{4}{|l|}{ Sputum Inflammatory Cells } \\
\hline Total Cell Count x $10^{5}$ cells $/ \mathrm{mL}$ & $45.6 \pm 61.2$ & $42.6 \pm 44.7$ & $45.9 \pm 53.6$ \\
\hline Neutrophils, \% & $45.3 \pm 23.8$ & $49.6 \pm 22.5$ & $45.5 \pm 27.8$ \\
\hline Macrophages, $\%$ & $24.5 \pm 14$ & $19.7 \pm 14.4$ & $23.3 \pm 15.0$ \\
\hline Eosinophils, \% & $1.7 \pm 2.5$ & $2.1 \pm 3.0$ & $0.6 \pm 0.9$ \\
\hline Lymphocytes, \% & $1.5 \pm 1.2$ & $1.4 \pm 1.1$ & $1.4 \pm 1.2$ \\
\hline AM Black Carbon, $\mu \mathrm{m}^{2}$ & $3.8 \pm 3.8$ & $4.7 \pm 4.0$ & $3.7 \pm 4.0$ \\
\hline
\end{tabular}

$\mathrm{SD}=$ standard deviation; $\mathrm{BMI}=$ body mass index; $\mathrm{ICS}=$ inhaled corticosteroids; $\mathrm{FEV}_{1}=$ forced expiratory volume in 1 second; $\mathrm{AM}=$ airway macrophages

$\mathrm{FEV}_{1} \%$ predicted of $60.8 \%( \pm 12.8)$ predicted. The first available sputum was from baseline, and 3 -and 6-month follow-up for 20, 5 and 5 participants, respectively. The samples that had adequate cell count for flow cytometry were obtained at baseline, and 3-and 6-month follow-up for 9, 3, and 4 participants, respectively. During the follow-up periods following sputum collection, 6 participants had 1 or more episodes of severe exacerbations, including 1 participant who reported 3 episodes during his/her time in the study. Fourteen participants had 1 or more episodes of any exacerbation (mild, moderate, or severe) during their time in the study following sputum collection and the characteristics of these 14 are shown in Table 1 . The average follow-up time following sputum collection was 189 days ( 6 months) and the annualized rate of exacerbations was 2.1 for any exacerbation and 0.6 for severe exacerbations. Two participants did not have the data for prospective risk of exacerbations. Sixteen patients (53\%) were using inhaled corticosteroids (Table 1) and no patients were on oral corticosteroids.

The mean $( \pm S D)$ total cell count in the sputum was $45.6 \times 10^{5}$ with $24.5 \% \pm 14$ macrophages (Table 1). The mean sputum black carbon area was $3.78 \mu \mathrm{m}^{2}$. An example of images of airway macrophages exhibiting 


\section{Figure 1. Digital Color Images of Airway Macrophages in Induced Sputum}

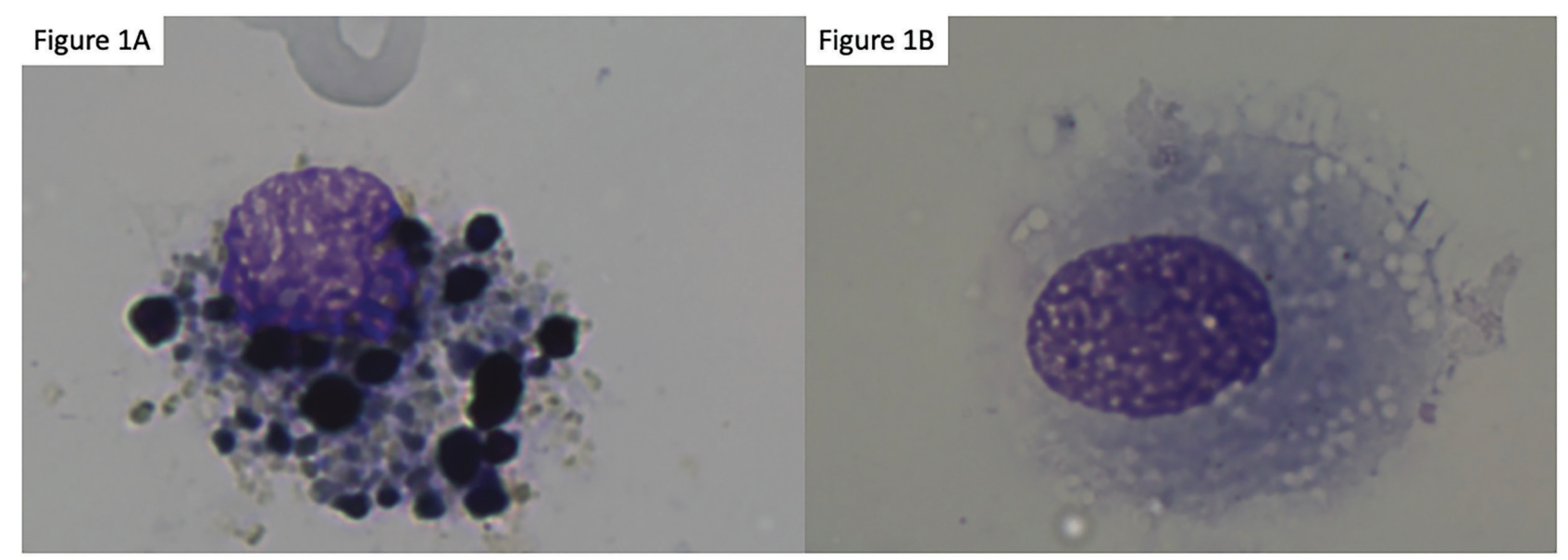

Image (a) is an example of an AM with high content of intracellular black carbon and Image (b) has no black carbon content.

$\mathrm{AM}=$ airway macrophage

high and no black carbon loading is shown (Figure 1).

\section{Association of Airway Black Carbon with COPD Outcomes}

After adjustment for covariates, higher airway black carbon content was associated with an increased rate of total exacerbations (incidence rate ratio $[\mathrm{IRR}]=1.20$ per $\mu \mathrm{m}^{2}$ increase, $p=0.032$ ) and severe exacerbations (IRR=1.31, $p=0.007)$, over the total follow-up period (Table 2). Similar results were identified in secondary analyses looking only at exacerbations in the 3 months following measurement. Airway black carbon content was not associated with other measured clinical outcomes: ECSC, FEV 1 , St George's Respiratory Questionnaire (SGRQ), CAT or mMRC, although directionally a higher black carbon content corresponded to worse outcomes for FEV $1, S G R Q$, and $\mathrm{mMRC}$ (Table 2). Percentage of black carbon positive macrophages trended toward higher total exacerbations (IRR=1.03 per percentage increase, $p=0.074$ ) and was associated with prospective severe exacerbations (IRR=1.05, $p=0.02$ ). Furthermore, percentage of black carbon positive was not associated with other clinical measures.

\section{Flow Cytometry}

There were no significant differences between the participants that did and did not have adequate cell counts for flow cytometry (Table 1). Increased black carbon content in airway macrophage was associated with reduced CD80 expression in the adjusted model $(\beta=-3.41, P=0.007)$ but not in the unadjusted model
( $\beta=-1.59, P=0.118)$, though Spearman rank correlation showed significant association in both unadjusted (rhospearman=-0.57, $P=0.019$ ) and adjusted models (rhospearman=-0.77, $P=0.009$ ) (Figure 2). However, with Bonferroni correction for multiple testing, these associations did not reach significance for adjusted models, $P=0.062$ ( $P_{\text {Spearman }}=0.072$ ), respectively. There was no association with black carbon content and CD274, HLA-DR, IL-1 $\beta$, IL-8, IL-10, TGF- $\beta 1$, IL-1Ra and TNF- $\alpha$ expression. The results were similar when using Spearman's rank correlations. Percentage of black carbon positive macrophages was associated with reduced $C D 80$ expression in the adjusted model $(\beta=-0.51, P=0.018)$, but not Spearman's rank correlation (rhospearman $=-0.22, P=0.2$ ).

\section{Discussion}

Particulate matter and biomass exposure have been independently associated with macrophage carbonaceous loading ${ }^{10,11}$ and in separate studies, with worse COPD morbidity. ${ }^{17,18}$ However, it has not been established if the AM black carbon influences COPD morbidity or macrophage function. Here, we report the novel finding of AM black carbon association with prospective total and severe exacerbations among former smokers with COPD. Given the deposition of this carbonaceous material in the macrophage, we hypothesized that it will skew macrophage phenotype leading to an abrogated immune response as the cause of increased 


\section{Table 2. Airway Macrophages Black Carbon Content Associations with COPD Outcomes ${ }^{a}$}

\begin{tabular}{|c|c|c|c|c|}
\hline & IRR & \multicolumn{2}{|c|}{$95 \% \mathrm{Cl}$} & $p$ \\
\hline Severe Exacerbations Over Total Follow-Up Period & 1.309 & 1.075 & 1.593 & 0.007 \\
\hline Any Exacerbation Over Total Follow-Up Period & 1.197 & 1.016 & 1.410 & 0.032 \\
\hline & $\beta$ & \multicolumn{2}{|c|}{$95 \% \mathrm{Cl}$} & $p$ \\
\hline Ease of Cough \& Sputum Clearance Score & 0.006 & -0.339 & 0.352 & 0.971 \\
\hline St George's Respiratory Questionnaire Total & 0.849 & -1.164 & 2.862 & 0.394 \\
\hline COPD Assessment Test & 0.092 & -0.684 & 0.868 & 0.809 \\
\hline modified Medical Research Council & 0.091 & -0.030 & 0.212 & 0.134 \\
\hline Post-bronchodilator FEV 1 \% Predicted & -1.093 & -2.329 & 0.144 & 0.081 \\
\hline
\end{tabular}

$\mathrm{a}_{\mathrm{n}=30}$; Adjusted by gender and inhaled corticosteroid use

$\mathrm{IRR}=$ incidence rate ratio; $\mathrm{CI}=$ confidence interval; $\mathrm{FEV}_{1}=$ forced expiratory volume in 1 second

\section{Figure 2. Black Carbon Content and Relation to CD80 Expression ${ }^{a}$}

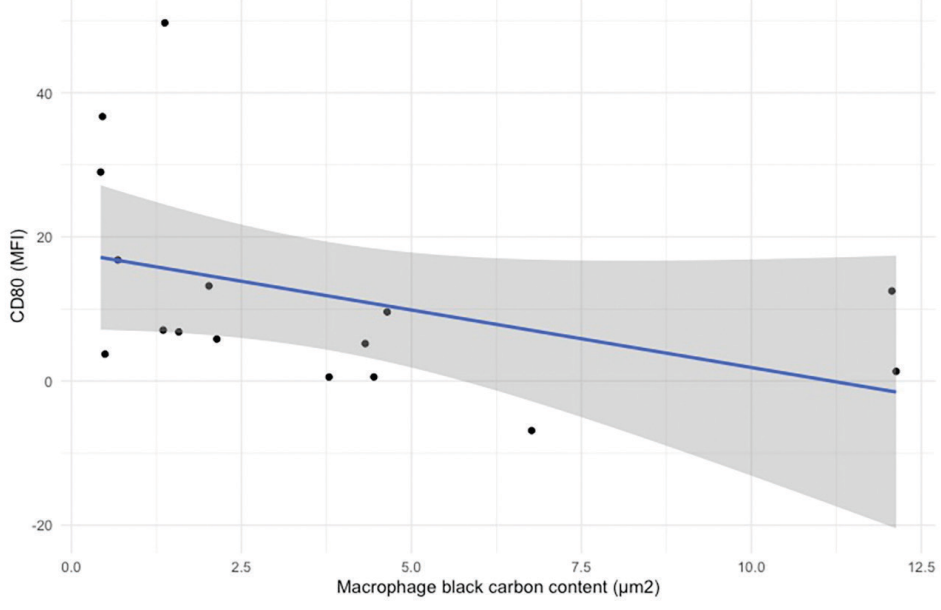

$\mathrm{a}_{\text {mean fluorescence intensity }}$

exacerbations. Through flow cytometry isolation of these cells, we identified the additional novel association of black carbon content with reduced AM CD80 expression proving a plausible mechanism for increased exacerbations.

Airway macrophage black carbon content is associated with environmental exposures, $10,11,19,20$ however, its impact on respiratory disease morbidity has been largely unexplored. We hypothesized that the AM black carbon content would be associated with worse morbidity given dysregulated macrophage function. In both the total follow-up period (an average of approximately 6 months) and the immediate next 3 months following assessment, increased AM black carbon content was associated with increased total and severe exacerbations. Although the exact lifespan of human AM is unknown, 6-month exposures are most strongly associated with AM black carbon content $^{20}$ and in a case report following bone marrow transplantation, patient's AM disappeared linearly, with their life span being approximately 81 days. ${ }^{21}$ Therefore, the exacerbations captured in our followup period could reflect the AM black carbon content we observed. In addition to the novel association of AM black carbon content with exacerbations, we identified directionally worse associations of AM black carbon content with other markers of COPD morbidity including a trend toward worse postbronchodilator $\mathrm{FEV}_{1} \%$ predicted $(\beta=-1.093, p=0.08)$ suggesting we may have been underpowered to identify statistically significant associations with these outcomes. The trend toward a reduced FEV 1 is consistent with what was identified by Kulkarni et al in a study of children with asthma. ${ }^{19}$ Notably, that study did not capture exacerbations and the association of AM black carbon content with exacerbations is previously unstudied. These findings have prompted interest in characterizing AM with increased black carbon content and speculation that their phagocytic ability may be impaired. ${ }^{22,23}$ We characterized these macrophages with the use of flow cytometry and identified AM black carbon content was associated with reduced CD80 expression.

The surface marker CD80 plays an important role providing costimulatory signals required for the development of antigen-specific immuneresponses. ${ }^{24}$ CD80 is also a marker for M1 macrophages which have enhanced phagocytic capability. ${ }^{25,26}$ Previous 
studies have demonstrated reduced macrophage CD80 expression in COPD participants compared to healthy volunteers. ${ }^{27,28}$ Further supporting the importance of CD80 in COPD, increased serum autoantibodies against CD80 have been associated with worse COPD stage. $^{29}$ Reduced CD80 can result in attenuated immunity, leading to a higher propensity for pathogen colonization which, in turn, is associated with advanced airway inflammation, increased frequency of exacerbation and accelerated decline in lung function. ${ }^{30-34}$

There are limitations of this study that merit discussion. Our analysis is associative in nature and although our findings suggest a plausible biologic model, this alone does not prove causation. Our sample size may be underpowered to detect the effect of AM black carbon content on other health outcomes, surface markers or intracellular cytokines. We utilized multiple surface markers and intracellular cytokines which poses the potential for spectral overlap, however, we sought to mitigate this with the use of FMO and compensation beads; compensation performed using both FACSDiva software and FlowJo demonstrated no spectral overlap for any of the fluorophores. Although sample availability precluded this, future studies could purify CD80+ macrophages and measure black carbon content to further eliminate the possibility of spectral overlap influencing our findings. Additionally, more thorough macrophage phenotyping with additional surface markers (e.g., CD 163, CD206) could be performed in future studies. We did not have a control group that would also help identify if this relationship is unique to COPD. Lastly, an FEV $1<35 \%$ predicted precludes safe performance of sputum induction and as such, this subset of more severe COPD patients was not included in our study. No participants in an exacerbation were enrolled which strengthens our findings given observations were made during steady state. Similar to others' studies of sputum cell count COPD in steady state reporting over $70 \%$ neutrophils, ${ }^{35,36}$ we observed a neutrophil predominance although less than these studies.

Our findings suggest that reduction in CD80 expression is the pathophysiologic mechanism for the association of AM black carbon content and increased exacerbations in former smokers with COPD. Therefore, beyond simply serving as a marker for increased exposures, AM black carbon content may be a predictor of future exacerbations given a macrophage less equipped to respond to an acute infectious exposure. This study furthers our understanding of the influence of AM black carbon content on COPD morbidity and macrophage phenotype.

\section{Acknowledgments}

Author Contributions: VT, KS, FD and $\mathrm{NNH}$ conceived of the presented idea. LG and KR recruited patients and performed sputum induction. VT, EM and ST performed black carbon quantification. VT, NA and FD performed flow cytometric and processed flow cytometry data. VT, KS, NP, JW, and $\mathrm{NNH}$ performed biostatistical analysis. All authors discussed the results and contributed to the final manuscript.

\section{Declaration of Interest}

Drs. Tejwani, Suresh, Putcha, Alexis, Moughames, Tang, Romero, Woo, D'Alessio and Ms. Grammer have nothing to disclose. Dr. Hansel reports grants and personal fees from AstraZeneca, grants and personal fees from GSK, grants from Boehringer Ingelheim, grants from the COPD Foundation, and personal fees from Mylan, all outside the submitted work. 


\section{References}

1. Lozano R, Naghavi M, Foreman K, et al. Global and regional mortality from 235 causes of death for 20 age groups in 1990 and 2010: a systematic analysis for the Global Burden of Disease Study 2010. Lancet. 2012;380(9859):2095-2128.

doi: https://doi.org/10.1016/s0140-6736(12)611728-0.

Erratum in: Lancet. 2013;381(9867):628.

2. Wynn TA, Chawla A, Pollard JW. Macrophage biology in development, homeostasis and disease. Nature. 2013;496:445-455. doi: https://doi.org/10.1038/nature12034

3. Vlahos R, Bozinovski S. Role of alveolar macrophages in chronic obstructive pulmonary disease. Front Immunol. 2014; 5:435. doi: https://doi.org/10.3389/fimmu.2014.00435

4. Yamasaki K, Van Eeden SF. Lung macrophage phenotypes and functional responses: role in the pathogenesis of COPD. Int J Mol Sci. 2018;19(2):582.

doi: https://doi.org/10.3390/ijms19020582

5. Löfdahl JM, Wahlström J, Sköld CM. Different inflammatory cell pattern and macrophage phenotype in chronic obstructive pulmonary disease patients, smokers and non-smokers. Clin Exp Immunol. 2006;145(3):428-437.

doi: https://doi.org/10.1111/j.1365-2249.2006.03154.x

6. Frankenberger M, Eder C, Hofer TPJ, et al. Chemokine expression by small sputum macrophages in COPD. Mol Med. 2011;17:762-770. doi: https://doi.org/10.2119/molmed.2010.00202

7. Alexis NE, Lay JC, Zeman KL, Geiser M, Kapp N, Bennett WD. In vivo particle uptake by airway macrophages in healthy volunteers. Am J Respir Cell Mol Biol. 2006;34(3):305-313.

doi: https://doi.org/10.1165/rcmb.2005-0373OC

8. Atkinson RW, Kang S, Anderson HR, Mills IC, Walton HA. Epidemiological time series studies of PM2.5 and daily mortality and hospital admissions: a systematic review and meta-analysis. Thorax. 2014;69(7):660-665.

doi: https://doi.org/10.1136/thoraxjnl-2013-204492

9. Dominici F, Peng RD, Bell ML, et al. Fine particulate air pollution and hospital admission for cardiovascular and respiratory diseases. JAMA. 2006;295(10):1127-1134.

doi: https://doi.org/10.1001/jama.295.10.1127

10. Fullerton DG, Jere K, Jambo K, et al. Domestic smoke exposure is associated with alveolar macrophage particulate load. Trop Med Int Heal. 2009;14(3):349-354.

doi: https://doi.org/10.1111/j.1365-3156.2009.02230.x

11. Belli AJ, Bose S, Aggarwal N, et al. Indoor particulate matter exposure is associated with increased black carbon content in airway macrophages of former smokers with COPD. Environ Res. 2016;150:398-402.

doi: https://doi.org/10.1016/j.envres.2016.06.025
12. Alexis N, Soukup J, Ghio A, Becker S. Sputum phagocytes from healthy individuals are functional and activated: A flow cytometric comparison with cells in bronchoalveolar lavage and peripheral blood. Clin Immunol. 2000; 97(1):21-32.

doi: https://doi.org/10.1006/clim.2000.4911

13. Jones PW, Harding G, Berry P, Wiklund I, Chen WH, Kline Leidy N. Development and first validation of the COPD Assessment Test. Eur Respir J. 2009; 34(3):648-654. doi: https://doi.org/10.1183/09031936.00102509

14. Mahler DA, Wells CK. Evaluation of clinical methods for rating dyspnea. Chest. 1988;93(3):580-586. doi: https://doi.org/10.1378/chest.93.3.580

15. Miller MR, Hankinson J, Brusasco V, et al. ATS/ERS Task Force: standardisation of spirometry. Eur Respir J. 2005; 26(2):319-338. doi: https://doi.org/10.1183/09031936.05.00034805

16. Lay JC, Peden DB, Alexis NE. Flow cytometry of sputum: assessing inflammation and immune response elements in the bronchial airways. Inhal Toxicol. 2011;23(7):392-406. doi: https://doi.org/10.3109/08958378.2011.575568

17. Hansel NN, McCormack MC, Belli AJ, et al. In-home air pollution is linked to respiratory morbidity in former smokers with chronic obstructive pulmonary disease. Am J Respir Crit Care Med. 2013;187(10):1085-1090.

doi: https://doi.org/10.1164/rccm.201211-1987OC

18. Miele CH, Grigsby MR, Siddharthan T, et al. Environmental exposures and systemic hypertension are risk factors for decline in lung function. Thorax. 2018;73(12):1120-1127. doi: https://doi.org/10.1136/thoraxjnl-2017-210477

19. Kulkarni N, Pierse N, Rushton L, Grigg J. Carbon in airway macrophages and lung function in children. $N$ Engl $J$ Med. 2006;355:21-30. doi: https://doi.org/10.1056/NEJMoa052972

20. Jacobs L, Emmerechts J, Mathieu C, et al. Air pollution-related prothrombotic changes in persons with diabetes. Environ Health Perspect. 2010;118(2):191-196. doi: https://doi.org/10.1289/ehp.0900942

21. Thomas ED, Ramberg RE, Sale GE, Sparkes RS, Golde DW. Direct evidence for a bone marrow origin of the alveolar macrophage in man. Science. 1976;192(4243):1016-1018. doi: https://doi.org/10.1126/science.775638

22. Brugha RE, Mushtaq N, Round T, et al. Carbon in airway macrophages from children with asthma. Thorax. 2014; 69(7): 654-659. doi: https://doi.org/10.1136/thoraxjnl-2013-204734

23. Lundborg M, Dahlén SE, Johard U, et al. Aggregates of ultrafine particles impair phagocytosis of microorganisms by human alveolar macrophages. Environ Res. 2006;100(2):197-204. doi: https://doi.org/10.1016/j.envres.2005.08.007 
24. Zhang P, Martin M, Yang QB, Michalek SM, Katz J. Role of B7 Costimulatory molecules in immune responses and T-Helper cell differentiation in response to recombinant HagB from porphyromonas gingivalis. Infect Immun. 2004;72(2):637-644. doi: https://doi.org/10.1128/IAI.72.2.637-644.2004

25. Lam RS, O’Brien-Simpson NM, Holden JA, Lenzo JC, Fong SB, Reynolds EC. Unprimed, M1 and M2 macrophages differentially interact with Porphyromonas gingivalis. PLoS One. 2016;11(7): e0158629. doi: https://doi.org/10.1371/journal.pone.0158629

26. Tarique AA, Logan J, Thomas E, Holt PG, Sly PD, Fantino E. Phenotypic,functional,and plasticity features of classical and alternatively activated human macrophages. Am J Respir Cell Mol Biol. 2015;53(5):676-688.

doi: https://doi.org/10.1165/rcmb.2015-0012OC

27. Pons AR, Noguera A, Blanquer D, Sauleda J, Pons J, Agustí AGN. Phenotypic characterisation of alveolar macrophages and peripheral blood monocytes in COPD. Eur Respir J. 2005;25(4):647-688. doi: https://doi.org/10.1183/09031936.05.00062304

28. Hodge S, Matthews G, Mukaro V, et al. Cigarette smoke-induced changes to alveolar macrophage phenotype and function are improved by treatment with procysteine. Am J Respir Cell Mol Biol. 2011;44(5):673-681.

doi: https://doi.org/10.1165/rcmb.2009-04590C

29. Luo XM, Liu XY, Tang JH, et al. Autoantibodies against CD80 in patients with COPD. Clin Transl Immunol. 2016;5:e102. doi: https://doi.org/10.1038/cti.2016.57

30. Sethi S, Murphy TF. Bacterial infection in chronic obstructive pulmonary disease in 2000:a state-of-the-art review. Clin Microbiol Rev. 2001;14(2): 336-363.

doi: https://doi.org/10.1128/CMR.14.2.336-363.2001

31. Bresser P, Out TA, Van Alphen L, Jansen HM, Lutter R. Airway inflammation in nonobstructive and obstructive chronic bronchitis with chronic Haemophilus influenzae airway infection: Comparison with noninfected patients with chronic obstructive pulmonary disease. Am J Respir Crit Care Med. 2000;162(3):947-952.

doi: https://doi.org/10.1164/ajrccm.162.3.9908103

32. Patel IS, Seemungal TAR, Wilks M, Lloyd-Owen SJ, Donaldson GC, Wedzicha JA. Relationship between bacterial colonisation and the frequency, character, and severity of COPD exacerbations. Thorax. 2002;57(9):759-764. doi: https://doi.org/10.1136/thorax.57.9.759

33. Hill AT, Campbell EJ, Hill SL, Bayley DL, Stockley RA. Association between airway bacterial load and markers of airway inflammation in patients with stable chronic bronchitis. Am J Med. 2000;190(4):288295. doi: https://doi.org/10.1016/S0002-9343(00)00507-6

34. Wilkinson TMA, Patel IS, Wilks M, Donaldson GC, WedzichaJA. Airway bacterial load and $\mathrm{FEV}_{1}$ decline in patients with chronic obstructive pulmonary disease. Am J Respir Crit Care Med. 2003;167(8):10961095. doi: https://doi.org/10.1164/rccm.200210-11790C
35. Singh D, Edwards L, Tal-Singer R, Rennard S. Sputum neutrophils as a biomarker in COPD: findings from the ECLIPSE study. Respir Res. 2010;11:77.

doi: https://doi.org/10.1186/1465-9921-11-77

36. Gao J, Zhou W, Chen B, Lin W, Wu S, Wu F. Sputum cell count: biomarkers in the differentiation of asthma, COPD and asthma-COPD overlap. Int J Chron Obstruct Pulmon Dis. 2017;12:2703-2710. doi: https://doi.org/10.2147/COPD.S142466 